\title{
Analyser la sécurité : Dillon, Waever, Williams et les autres
}

Ayse Ceyhan

\section{OpenEdition}

1 Journals

Édition électronique

URL : http://journals.openedition.org/conflits/541

DOI : $10.4000 /$ conflits.541

ISSN : $1777-5345$

Éditeur :

CCLS - Centre d'études sur les conflits lilberté et sécurité, L'Harmattan

Édition imprimée

Date de publication : 15 mai 1998

ISSN : 1157-996X

\section{Référence électronique}

Ayse Ceyhan, "Analyser la sécurité : Dillon, Waever, Williams et les autres », Cultures \& Conflits [En

ligne], 31-32 I printemps-été 1998, mis en ligne le 16 mars 2006, consulté le 30 mars 2021. URL:

http://journals.openedition.org/conflits/541 ; DOI : https://doi.org/10.4000/conflits.541

Ce document a été généré automatiquement le 30 mars 2021.

Creative Commons License 


\title{
Analyser la sécurité : Dillon, Waever, Williams et les autres
}

\author{
Ayse Ceyhan
}

1 La notion de sécurité est omniprésente dans les préoccupations quotidiennes des individus. Elle touche pratiquement tous les aspects de la vie. On parle de la sécurité de l'individu, de la sécurité nationale, de la sécurité sociale, de la sécurité aérienne, de la sécurité routière, de la sécurité alimentaire, etc. On évoque le besoin de sécurité, le désir de sécurité. Mais, en même temps, la notion de sécurité parait si évidente que l'on ne se donne pas la peine de s'interroger sur elle. Elle est là, elle va de soi. Or, comme le dit Arthur Koestler, les choses les plus évidentes sont celles qui méritent le plus d'être examinées. Aussi, la sécurité nécessite-elle d'être examinée minutieusement. Se pose alors la question de son analyse. Comment faut-il l'appréhender ? Faut-il s'interroger sur la bonne définition, sur le(s) objet(s) référent(s), sur le(s) secteur(s), les menaces? Faut-il se préoccuper des pratiques et des techniques de sécurisation? Faut-il analyser la sécurité sous son rapport au politique?

2 Pendant longtemps sous l'influence de la vision réaliste du monde, la réponse qui emporta l'adhésion quasi totale de tous a été de concevoir la sécurité comme une question de survie et de lui présupposer une menace existentielle. On l'appréhenda essentiellement comme ayant trait à la survie physique de l'Etat-nation et à l'agression armée potentielle contre lui. Réduite ainsi à une question de protection et de défense, elle fut considérée comme constituant l'objet privilégié des études de la défense et de la stratégie. Sous l'emprise de cette approche, les analyses en relations internationales se sont cantonnées à appréhender la sécurité plus comme un objet empirique que comme un concept. Il y a eu certes les travaux de Wolfers, de Herz et de Laswell ${ }^{1}$. Mais, en général, la sécurité a été considérée comme un objet méritant plus un traitement empirique que réflexif. Ce choix s'explique également par la fusion effectuée par le réalisme entre les notions de sécurité et de puissance. La sécurité a été considérée comme un dérivé de la puissance et a été reléguée au statut d'indicateur de succès des Etats dans la compétition militaire. Dès lors, les analyses portant sur elle se sont réduites à une cartographie de la puissance et des menaces militaires ${ }^{2}$. Privilégiant 
ainsi la perspective empirique, ces analyses ont cependant négligé une dimension fondamentale de la sécurité : sa dimension ontologique et épistémologique. Comme il ressort des écrits de Hobbes, de Descartes et de Heidegger, la sécurité a trait à l'Etre, à l'existence, à la connaissance, à la relation aux autres êtres humains, à la médiation entre la vie et la mort, à la médiation entre le chaos et l'ordre. Dans son livre Politics of Security, Michaël Dillon analyse cette dimension en affirmant que la conception de la sécurité dérivée de la vision hobbesienne de la nature humaine et du politique repose en fait sur la tradition métaphysique qui fait d'elle le principe déterminant, l'archè de la vie et de la politique moderne ${ }^{3}$. Selon cette tradition, posée de façon axiomatique comme un principe d'auto-préservation, de droit à la vie, à la survie ainsi que comme droit à la vérité, la sécurité est intrinsèquement liée au savoir, à la certitude, à la rationalité, à la calculabilité, à la technologie. Dillon affirme que si la sécurité a ainsi constitué le fondement de la pensée politique moderne, la tâche de la pratique politique a été de " sécuriser la sécurité "4 en instrumentalisant la technologie. Celle-ci n'a été conçue que dans le cadre de l'Etat, sans quoi et en dehors de quoi la métaphysique occidentale a été incapable de concevoir la sécurité. Cependant, si cette tradition a été maintenue pendant la période d'après-guerre et pendant la bipolarité, depuis la fin des années soixante-dix, les phénomènes de mondialisation, de transnationalisation, de fragmentation, l'émergence des réseaux, les diasporas, etc. ont donné lieu à une nouvelle lecture du monde. Les thèmes du chaos, du désordre, de l'hétérogénéité, du clash civilisationnel, de la balkanisation énoncés dans le discours des néoréalistes ont remplacé l'idée de l'ordre et de la certitude introduisant la question du doute, de la peur épistémologique, de l'incertitude 5 . Sur le plan de l'analyse, ceci a donné lieu à une remise en cause de l'approche classique. La question qui se posait alors était de savoir si on pouvait continuer à concevoir la sécurité comme une question de défense et de stratégie. Dès le début des années quatre-vingt les internationalistes ont commencé à s'interroger sur les objets référents, les secteurs et les dimensions de la sécurité. Fallait-il les approfondir, les élargir?

C'est à Barry Buzan que l'on doit une interrogation sur les relations entre sécurité et défense ainsi qu'une tentative de théorisation de la question de la sécurité. Dans son livre People, States and Fear, publié initialement en $1983^{6}$, Buzan a affirmé que le sécurité ne se réduisait pas à la seule sécurité nationale (ou la sécurité de l'Etat), mais qu'elle s'élargissait à de nouveaux objets et secteurs. Ces secteurs sont le militaire, le politique, l'économique, l'environnemental et le sociétal. Cependant, l'analyse de Buzan ne se limitait pas à décrire ce phénomène, mais elle tentait aussi de l'expliquer. Pour ce faire, il semblait affirmer que l'élargissement de la sécurité était le résultat de l'apparition de nouvelles menaces " objectives " contre les sociétés occidentales. Même si, dans la deuxième édition de son livre en 1991, Buzan restait plus prudent et parlait des logiques sectorielles donnant lieu à des productions sécuritaires, il continuait à reproduire l'idée que la sécurité signifie la survie et présuppose une menace existentielle. Au fond, comme le note Didier Bigo, en séparant la sécurité subjective et la sécurité objective, l'analyse de Buzan revient à distinguer entre " vraies " et " fausses " menaces et ne se démarque pas vraiment de la vision réaliste faisant de l'Etat son objet référent et des gouvernements ses acteurs centraux ${ }^{7}$. Cette distinction constitue un des pièges dans lesquels tombent facilement les internationalistes contemporains. Que faire pour l'éviter? Comment éliminer des analyses toute possibilité de référence à l'objectivation de la menace ? Tâche difficile qui, comme nous le verrons, nécessite une révision des méthodes et approches utilisées jusqu'à présent. Mais il faut reconnaître 
que la brèche ouverte par Buzan a permis aux internationalistes d'élargir l'agenda des études de la sécurité à de nouveaux secteurs (économique, écologique, démographique, identitaire, etc.) et de l'approfondir en introduisant de nouveaux objets référents comme l'international, le régional, le local, ou bien comme la société, la nation, la communauté, le groupe, l'individu, etc. ${ }^{8}$.

4 A présent, nombreux sont les travaux qui se proposent de forger une approche postréaliste, poststructuraliste, postmoderne de la sécurité. Parmi eux, on peut distinguer les analyses d'Ole Waever et de ce qui est communément appelé l'école (ou le groupe ?) de Copenhague, celles de Michaël Dillon et celles des auteurs réunis sous le nom de Critical Security Studies (études critiques de la sécurité) ${ }^{9}$. En se référant aux travaux de Barry Buzan, Ole Waever poursuit son analyse en développant un nouvel objet référent (la sécurité sociétale) et en adoptant une approche constructiviste et linguistique. En s'inspirant des travaux de Heidegger, Michaël Dillon soumet l'analyse de la sécurité à une lecture double, inséparablement philosophique et politique. Quant aux auteurs réunis sous l'appellation Critical Security Studies, ils appartiennent à des horizons différents, allant du néoréalisme (Mohammed Ayoob) au constructivisme modéré (Beverly Crawford, Karin Fierke et Thomas Risse-Kappen) en passant par le postmodernisme (Ronnie D. Lipshutz, Michaël Williams, Keith Krauze, Simon Dalby, R. B. J. Walker, Ken Booth). Ils déclarent vouloir rompre avec la vision classique de la sécurité et introduire un large éventail de thèmes comme les droits de l'homme, le développement, les gender studies, l'émancipation, etc. et s'interrogent sur les rapports entre la sécurité et l'ordre politique qui lui correspond ${ }^{10}$. Aussi différents soient-ils, ces auteurs partagent un certain nombre de points communs dans leurs analyses. Ils se proposent de transformer l'approche instrumentale de la sécurité en une approche réflexive. Pour eux, la sécurité n'est pas le contraire de l'insécurité. Ils refusent de la considérer comme une donnée a priori et veulent la dé-essentialiser. Ils examinent (de différentes façons) son processus de construction et de transformation. Pour cela, ils l'appréhendent autant comme concept que comme pratique ${ }^{11}$. Cependant, il est nécessaire de noter que, malgré ces ressemblances, leurs analyses relèvent d'approches différentes et ne partagent pas les mêmes présupposés normatifs. Pour Waever, la sécurité est un concept auto-référentiel, pour Williams et Krause, ainsi que pour les auteurs de Critical Security Studies, c'est un concept dérivé, sans signification en soi, dépendant d'un objet et de la signification qui lui est attribuée alors que pour Dillon elle a trait à l'ontologie.

Qu'entendre par sécurité ? Définition, conceptualisation, étymologie, généalogie

Dans sa contribution au Critical Security Studies, Simon Dalby reprend la formule employée par Buzan pour caractériser le concept de sécurité comme un " essentially contested concept " (concept essentiellement contesté) ${ }^{12}$. Cette expression empruntée à la linguistique signifie que le concept de sécurité est l'objet de contestations et revêt plusieurs significations qui ne sont pas nécessairement liées à la définition conventionnelle, mais qui sont tributaires des conceptions politiques ${ }^{13}$. Elle implique que les modifications ou reformulations du concept peuvent être interprétées comme une contestation de fond. Cette expression est également reprise par Ole Waever qui note que " la sécurité est un concept essentiellement contesté qui ne peut pas être défini de façon précise en raison de son caractère politique inhérent ${ }^{14}$ ". Or, il faut rappeler que si Buzan avait émis des réserves quant au concept de sécurité, il avait 
quand même adopté une définition claire et nette de la sécurité comme " survie ou libération à l'égard des menaces " et avait bâti sa problématique à partir d'elle.

7 La question de la signification de la sécurité est de nouveau posée depuis l'effort d'élargissement et d'approfondissement de l'agenda des études de la sécurité. Pour certains, analyser le sens de la sécurité revient à la définir, c'est-à-dire à lui donner une signification condensée dans un énoncé. Pour d'autres, comme Ole Waever, il faut plutôt la conceptualiser, c'est-à-dire lui donner un contenu spécifique plus large et plus complexe, communicable par le langage. Selon Jef Huysmans, ces deux formes, qui ne se diffèrent pas quant à la signification du mot, sont au fond des " rites de passage " ou des " rituels de purification "15 courants dans le champ académique, qui peuvent donner lieu à des approches différentes pour établir le contenu de la sécurité. En présupposant une seule signification, la définition localise la recherche dans un registre particulier. La conceptualisation explore plus en détail ce qui caractérise une politique de sécurité ainsi que le débat qu'elle engendre. Elle permet au chercheur de partir d'un dénominateur commun pour construire un schéma analytique qui rend plus explicite ce que devrait être une analyse de la sécurité ${ }^{16}$. Sans entrer dans le débat définition ou conceptualisation, Michaël Dillon se propose d'examiner l'étymologie et la généalogie du terme. L'étymologie attire l'attention sur l'ordre du discours et sur ses termes essentiels en rappelant leur sens véritable. La généalogie s'interroge sur l'entrée de la sécurité dans le discours ${ }^{17}$. Pour Dillon, la sécurité est un terme dual qui signifie non seulement un moyen de libération à l'égard du danger, mais aussi un moyen de le contraindre, de le limiter ${ }^{18}$. Puisque la sécurité est engendrée par la peur, elle nécessite des contre-mesures pour contrôler, contenir, éliminer, neutraliser cette peur. Ainsi, tout en nous apprenant de quoi il faut avoir peur, elle cherche aussi à proscrire, à sanctionner, à punir, en quelque sorte à mettre en danger ce qui nous menace ${ }^{19}$. Cette double signification de la sécurité existait dès l'Antiquité, où elle était représentée par la figure duale de Poseïdon, à la fois dieu de la mer qui sécurisait et stabilisait et celui par qui arrivait un tremblement de terre, celui qui détruisait. Terme ambigu, la sécurité contient donc à la fois sécurité et insécurité que Dillon, en bon heideggerien, transcrit par le terme (in)sécurité ${ }^{20}$. Cette dualité est exprimée dans ses origines grecques, par le terme asphaleia, qui signifie sécurité, certitude et sûreté et qui est le dérivé de sphallo, qui signifie faire trébucher, se tromper, tomber. Quant à ses origines latines, le terme sécurité, dérivé de securitas, lui même dérivé de sine cura (sans inquiétude, sans anxiété, etc.), signifie non seulement libération à l'égard des soucis, à l'égard du danger, assurance, exactitude, mais aussi négligence, indifférence, insouciance ${ }^{21}$. Cependant, cette double signification ne relève pas seulement de la mythologie. On la retrouve également de nos jours quand on consulte, par exemple, l'Oxford English Dictionnary, qui attribue au terme deux types de significations. La première est centrée sur les conditions d'être en sécurité, la seconde a trait aux moyens de le devenir. Ceci pour montrer que la sécurité est liée à de nombreux thèmes comme la vérité, la certitude, l'absence d'anxiété, la contrainte, à la technologie, et est, en même temps, intimement liée à l'insécurité. En analysant ainsi l'étymologie et la généalogie du terme, Dillon veut montrer que la sécurité ne relève pas d'un fondement stable, mais qu'elle trahit sa propre essence en recherchant la stabilité, la certitude, la vérité. $\mathrm{Au}$ fond, dit-il, ce qui cause l'insécurité ce n'est pas l'idée de la mort ou une forme de vie particulière, mais l'(in)sécurité de l'existence elle-même ${ }^{22}$. On pourrait se demander ici, avec James Der Derian, comment on est passé de cette signification ambiguë, voire même incertaine, à une conception essentialiste de la sécurité ${ }^{23}$. La 
réponse nécessite peut-être d'approfondir l'analyse en intégrant d'autres facteurs, tels que la religion, les rapports entre le religieux et le politique, etc. Si pour Dillon il importe de rechercher les origines de la sécurité et de s'interroger sur sa généalogie, pour Waever il s'agit de procéder par un travail de conceptualisation. Ici, il nous faudrait interroger ce qu'il entend par conceptualisation. Si on considère son travail dans son ensemble, on remarque qu'il aborde la question de deux façons différentes. Il semble que dans ses premiers travaux ce qui l'intéresse est de forger une nouvelle conception de la sécurité pour analyser les préoccupations sécuritaires contemporaines. D'où son concept de la sécurité sociétale. Dans ses travaux ultérieurs, il se distingue d'une approche qui se focalise sur l'objet référent et propose de s'intéresser à la sécurité en elle-même, de l'appréhender comme un concept autoréférentiel ${ }^{24}$, de faire une " conceptualisation sécurisante de la sécurité " (securytiness of security) ${ }^{25}$. Pour cela, il intègre une dimension linguistique à son analyse et s'intéresse à la pratique discursive. En procédant ainsi, l'objectif de Waever est de sortir $\mathrm{du}$ piège vraie/fausse menace dans lequel il tombe dans ses premiers travaux. Il veut montrer que la sécurité ne se réfère pas à une réalité externe, objective, mais qu'elle établit une situation de sécurité par elle-même. Nous allons d'abord examiner sa première approche, montrer les ambiguïtés de celle-ci et analyser ensuite la seconde qui met en avant une dimension discursive.

8 La sécurité sociétale : une tentative de conceptualisation

9 Selon Waever, depuis la fin de la bipolarité, avec les phénomènes de mondialisation, de transnationalisation, de construction européenne et d'émergence des ethnonationalismes en Europe de l'Est, c'est la société plus que l'Etat qui est menacée. Dorénavant ce seraient plutôt les peurs liées à l'insécurité, aux incivilités, à l'Autre, à l'immigration, à l'invasion, à la perte des valeurs culturelles et des styles de vie etc. qui préoccuperaient les individus. Les attaques xénophobes contre les demandeurs d'asile en Allemagne, le " non " danois au Traité de Maastricht, la purification ethnique en exYougoslavie seraient des exemples qui ne feraient que confirmer ces peurs. A ses yeux, pour analyser ces nouveaux enjeux, le concept de sécurité sociétale apparaît comme le concept théorique cohérent et pertinent ${ }^{26}$. Waever a développé sa conception de la sécurité sociétale en s'inspirant des notions initialement introduites par Buzan ${ }^{27}$. Reprenant les catégories développées par celui-ci, il les a ré-agrégées en deux : la sécurité nationale et la sécurité sociétale. Selon lui, la première a trait à la souveraineté et à la survie du régime, et la seconde concerne l'identité et à la survie de la société. En centrant son analyse sur la sécurité sociétale, il s'est proposé de déplacer l'objet référent de la sécurité de l'Etat vers la société et d'élever ce dernier au statut d'objet indépendant. Présentant la sécurité sociétale comme " la capacité d'une société à persister dans ses caractéristiques essentielles face aux conditions changeantes et face à des menaces probables ou réelles "28, Waever affirme que celle-ci a trait à la survie de la société. Ici, il nous faut indiquer que lorsqu'il parle de la société, il s'agit moins de sa structure, de son organisation que de son identité. Pour lui, " la société touche à la notion d'identité, à l'idée que des groupes et des individus se font d'eux-mêmes et qui leur permet de s'identifier comme membres d'une communauté "29. Les préoccupations sécuritaires contemporaines porteraient alors sur la préservation de l'identité et de la défense de la culture. Ce qu'il est important de souligner ici, c'est qu'en adoptant le concept de sécurité sociétale, on transforme l'identité et la culture en un enjeu sécuritaire. Elles sont présentées comme une question de survie, comme quelque chose à défendre face à un danger, à une menace. Aussi novateur soit-il, le concept de sécurité 
sociétale présente néanmoins quelques ambiguïtés. D'abord sur le plan de la sémantique, le terme générique " sociétal " ne paraît pas très compréhensible. Pourquoi " sociétal " ? Waever affirme qu'il a maintenu la dénomination originelle fournie par Buzan ${ }^{30}$. Il reconnaît lui-même qu'idéalement le terme devrait être celui de " sécurité identitaire ". Il y a aussi une ambiguïté de fond quant à l'objet même du concept. S'agit-il de la société, de la nation ou de la communauté ? On ne comprend pas exactement la nature de cet objet référent. On a l'impression que la société et la nation se confondent. La société au lieu d'être appréhendée comme un lieu d'interactions sociales complexes et de conflits, apparaît comme étant confondue avec la nation, acceptée au sens de communauté identitaire, représentant une culture et un mode de vie particuliers qu'il faut absolument protéger. Pour développer cette acceptation, parmi ses références théoriques, Waever semble plutôt s'inspirer du romantisme et de l'idéalisme allemands dont l'un des inspirateurs les plus connus est Herder ${ }^{31}$. Quant à l'identité, elle apparaît plutôt comme quelque chose de statique, un donné. Le processus de sa (re)production, de (dé)formation n'est pas exploré. Même si ce n'est pas l'intention de Waever, son concept de sécurité sociétale risque d'aller dans le sens d'une essentialisation de l'identité sous forme d'objet naturel.

On peut dire que l'analyse de Waever oscille entre le descriptif et le justificatif. C'est comme si la sécurité sociétale se justifiait en tant qu'atteinte à l'identité. On ne saisit pas très bien l'aspect de construction de la menace. Nous dirons ici avec Didier Bigo, qu'il faut être prudent dans la manipulation de ce concept. S'il est bien utilisé il peut permettre l'analyse des opinions publiques. Mais pour cela il faudra procéder à une analyse sociologique où il sera nécessaire d'identifier les acteurs qui énoncent la sécurisation des thèmes d'identité et de culture. Il faudra analyser leur position sociale, les jeux de pouvoir et de domination, les luttes entre agents et politiciens pour imposer une définition légitime de la menace, etc. Considéré dans sa formulation waeverienne, il est susceptible d'être instrumentalisé par les acteurs qui souhaitent mener une politique identitaire ${ }^{32}$. Enfin, concernant la distinction qu'il propose entre l'Etat et la société comme objets référents, il nous faudra noter que cette distinction n'apparaît pas dans les discours et les pratiques des agences de sécurité, pas plus que dans ceux des politiciens et des bureaucrates. Il est difficile de prétendre que la sécurité sociétale constitue leur seul objet focal. En effet, quand on les analyse on remarque qu'au lieu d'être séparés l'Etat et la société sont liés et, que les principales références sont l'Etat, la souveraineté et la frontière ${ }^{33}$. Et cette dernière qui apparaît comme le lieu clé qui délimite les pratiques des agences de sécurité n'apparaît guère dans l'analyse de Waever. Mais dans ses travaux ultérieurs, comme Securitization and Desecuritisation . ${ }^{34}$, Waever mettra de côté cette distinction entre la sécurité sociétale et la sécurité de l'Etat et orientera son analyse vers la construction discursive des menaces. Dorénavant, ce qui l'intéresse c'est l'analyse du processus discursif sécurisation qui prend comme référence les éléments touchant aux identités.

12 Afin de montrer que la sécurité ne se réfère pas à une réalité externe, objective, mais qu'elle établit une situation de sécurité par elle-même, Waever affirme qu'elle se réalise par le discours. Elle est construite par les caractéristiques propres au discours sécuritaire. C'est par l'acte de discours, le speech act, qu'un problème devient un problème de sécurité. En faisant ainsi, Waever pense dé-essentialiser la sécurité, c'està-dire ne pas la considérer comme quelque chose ou un état qui existe avant le 
discours ${ }^{35}$. Il veut montrer que la sécurité est une pratique auto-référentielle, que sa condition d'existence est constituée par le speech act lui-même, et non par une quelconque imposition d'une menace. Pour donner une dimension linguistique au concept de sécurisation, Waever s'inspire de la philosophie du langage et plus particulièrement des travaux d'Austin et de Derrida. Pour cela, il s'appuie explicitement sur la notion de speech act ou acte de langage. Une des notions essentielles de la pragmatique linguistique, le speech act signifie la plus petite unité réalisant une action (ordre, requête, assertion, promesse) par le langage ${ }^{36}$. Parmi les actes de langage, Waever s'intéresse plus particulièrement à la notion du performatif développée par Austin. Dérivé du mot anglais " to perform ", le performatif indique que produire l'énonciation (dire) c'est exécuter une action (faire) ${ }^{37}$. Prononcer, appeler, dire, annoncer, promettre, jurer, etc., sont ainsi des formes énonciatives constitutives de l'acte. Ainsi selon Waever, dans le processus de sécurisation, le mot " sécurité " n'est pas intéressant comme signe se référant à un objet concret qui existe déjà, mais c'est son énonciation qui constitue l'acte. C'est elle qui est la réalité première, elle produit ou transforme une situation. Elle opère. Aussi, un problème devient un problème de sécurité quand les responsables étatiques l'appellent ainsi. Ce faisant, ils modifient la nature du problème et élèvent les enjeux en high politics en leur conférant un caractère d'urgence et de nécessité. Ceci leur permet de prendre des mesures exceptionnelles ${ }^{38}$. Le discours sécuritaire est donc un acte de langage, une labellisation dotée d'une force illocutoire. L'intérêt d'une telle démarche est de focaliser l'attention sur les règles d'énonciation et de montrer la force de la labellisation comme forme de pouvoir symbolique.

13 Cependant, même si Waever parle des producteurs des énoncés et évoque parfois les contextes (comme l'européanisation ou la fin de la bipolarité) dans lesquels se produisent les discours, ceux-ci ne semblent pas très importants pour son analyse. Ce sont les qualités intrinsèques de l'acte de parole qui lui paraissent déterminantes plutôt que la position de ceux qui énoncent les discours. D'ailleurs, quand il évoque ces derniers il les désigne souvent en des termes globaux comme " les représentants de l'Etat "39 ou " certains groupes ou l'élite " 40 mais ne recourt pas à une analyse sociologique de leur position sociale, de leurs interactions, de leurs stratégies de mobilisation, de positionnement, de leurs luttes et coopération, etc. Son analyse relève plutôt d'une linguistique de l'énonciation ${ }^{41}$ qui étudie des objets décontextualisés et qui replie le langage sur l'arbitraire des règles, sur les propriétés régulières et sur les processus formels que d'une analyse qui met en avant les conditions sociales de la production des discours.

On peut dire que le penchant de Waever pour une linguistique de l'énonciation vient d'une lecture derridienne des travaux d'Austin. S'il se réfère explicitement à ce dernier ${ }^{42}$, il semble plutôt s'appuyer sur les interprétations de Derrida en empruntant par exemple à Austin la notion de la performativité sans toutefois procéder à une réelle analyse de la pragmatique linguistique qu'il a développée. Cette touche derridienne apparaît également explicitement dans la présentation de ses sources bibliographiques. Souvent, lorsqu'il cite le livre d'Austin How to do Things With Words, il lui adjoint le texte de Derrida publié en 1979 dans la revue $\mathrm{Glyph}^{43}$, où ce dernier a livré un commentaire de la théorie des actes de langage d'Austin. Or cet article a été vivement critiqué par John Searle ${ }^{44}$, ancien étudiant d'Austin, théoricien des actes de langage et continuateur de la théorie de l'illocutoire développée par ce dernier, disparu prématurément en 1960. Par revues interposées, Searle et Derrida se sont livrés à une 
querelle autour de l'interprétation des thèses d'Austin. Searle a reproché à Derrida d'avoir mal lu Austin et d'avoir fait une série de confusions et d'erreurs sur les concepts qu'il avait développés ${ }^{45}$. Derrida prend d'Austin le concept de la performativité mais il lui reproche de tenir compte du contexte réel ou linguistique de production des énoncés ${ }^{46}$. Les performatifs l'intéressent parce qu'il les considère comme produisant des effets, ayant valeur de force, de différence et échappant à l'autorité de la valeur de vérité, à la dichotomie du vrai et du faux ${ }^{47}$, qui constitue à ses yeux une opposition qu'il faut absolument miner. Mais il pense que la raison des échecs qui peuvent affecter les performatifs revient à l'insistance d'Austin sur le contexte. Pour lui le contexte est lié à la conscience, à la présence consciente de l'intention, facteurs qui empêchent de construire une théorie du langage comme un objet naturel et autonome. Au fond, pour Derrida, indépendamment du contexte et des intentions du producteur, ce qui est important c'est de mettre en avant le caractère " itérable " (répétable) du performatif. Il affirme qu'il y a une coupure entre le producteur d'un énoncé et le destinataire. Les énoncés se prêtent à la répétition. Ils peuvent être cités et rompre avec tout contexte donné. Il ne faut pas les considérer comme étant commandés par les intentions de l'énonciateur. Il est par ailleurs inutile de se soucier de la position sociologique de ce dernier. Bref, pour Derrida, l'analyse contextuelle est insuffisante et inutile ${ }^{48}$. En critiquant Austin, Derrida veut en fait revenir sur sa propre thèse destinée à miner l'opposition écrit/oral et à montrer qu'il n'existe rien en dehors du texte, que l'écriture est une vérité première et que la parole est en réalité une forme d'écriture (archiécriture). Searle reproche à Derrida l'usage qu'il fait du concept d'écriture dans le contexte d'une réflexion sur l'illocutoire. Il remet en question le lien privilégié qu'il établit entre les concepts " d'itérabilité ", de citationnalité et celui d'écriture. Contrairement à Derrida qui propose d'ignorer le contexte, il affirme que l'intentionnalité ne disparaît pas, mais qu'elle apparaît au contraire dans les compréhensions $d u$ sens $^{49}$. Il insiste pour que le rôle du contexte soit absolument explicité. Il propose de s'intéresser aux circonstances dans lesquelles les actes de langage se réalisent. Il met l'accent sur les règles qui régissent les actes et sur leur dimension institutionnelle. Selon lui, l'entreprise derridienne s'inscrit au fond dans la stratégie déconstructionniste qui consiste à miner les oppositions binaires traditionnelles comme parole/écriture, vérité/fiction, réalité/apparence, masculin/ féminin, etc. Ce qui revient à miner le " logocentrisme ", terme que Derrida emploie pour désigner l'intérêt pour la vérité, la rationalité, la logique et le " mot " qui marque la tradition philosophique occidentale ${ }^{50}$. Mais en voulant à tout prix défendre son approche, Derrida se place dans une position ambiguë. Il ramène tout à l'écriture et centre son analyse sur les traits, les marques, les signes, les " graphèmes ", les caractéristiques qu'on peut reconnaître dans le concept d'écriture. En faisant ainsi, il ne parvient pas véritablement à éliminer de sa pensée les oppositions, hiérarchies, exclusions, négations présumées du logocentrisme sur lesquelles porte l'entreprise critique du déconstructionisme ${ }^{51}$. Il faut également rappeler que Derrida a hérité de l'influence de Saussure. Ceci lui permet de ne pas identifier le langage à des substances ou à des événements physiques, mais à des formes. Pour lui, un signe linguistique ne se définit pas par un contenu substantiel mais par des différences. Lecteur de Derrida, Ole Waever semble lui aussi ne pas s'intéresser au contenu substantiel, et, comme nous l'avons déjà mentionné, ce sont les notions de performativité et d'illocutoire qui lui apparaissent déterminantes. Mais cette démarche, aussi intéressante soit-elle, appelle quelques remarques. 
15 Afin de pouvoir dépasser l'opposition vraie menace/fausse menace, Waever s'intéresse aux performatifs qui sont indifférents à la vérité ou à la fausseté. Ce qui est important c'est l'effet immédiat qu'ils produisent sur un auditoire. Cet aspect peut expliquer la raison pour laquelle il n'évoque pas une autre catégorie d'actes de langage développée par Austin, à savoir les constatifs. Ceux-ci se réfèrent aux faits et appartiennent à l'ordre de la représentation du monde et peuvent être caractérisés en termes de vérité et de fausseté. Or, ce qui semble intéresser Waever, c'est l'effet d'une technique de discours. Mais s'agissant de la sécurisation, la simple énonciation du mot " sécurité " ou du mot " menace " et leur répétition même à l'infini suffisent-elles à convaincre des personnes ou des groupes et à les mobiliser? Il convient de remarquer que l'effet dont il est question, c'est-à-dire persuader et convaincre, n'est pas que linguistique, simple conséquence de l'énonciation. Il se réalise aussi par la narration, l'argumentation, et la communication. Les discours ne sont pas que des énoncés et ne se réduisent pas à la seule production d'effet. Ils sont aussi des narratifs faisant référence à des événements réels ou imaginaires et des argumentatifs construisant un ordre de raisons. La démarche de Waever ne nous indique pas comment les discours sécuritaires se constituent du point de vue de leur argumentaire et comment ils structurent des opinions publiques.

16 En fait, quand on analyse les discours sécuritaires, on voit qu'il ne s'agit pas seulement de simples énoncés, mais d'un ensemble d'arguments rhétoriques qui sont déployés différemment en fonction des contextes (historiques ou présents) et des auteurs qui les développent. Ces arguments sont employés dans le but de modifier ou de renforcer par le biais du langage les représentations, croyances, valeurs d'un groupe ou d'une communauté. Ils peuvent être des arguments-types au sens où l'emploie Albert Hirschman ${ }^{52}$, comme la perte de la souveraineté, la corrélation entre l'immigration, le chômage, la criminalité et le trafic de drogue, l'inassimilabilité des migrants originaires des pays du Sud, etc., que l'on retrouve pratiquement dans tous les discours sécuritaires contemporains ${ }^{53}$. Ils peuvent également être inventés en fonction des besoins du moment, comme la crainte du multiculturalisme, les incivilités ou l'insécurité. Il convient de se demander pourquoi à un moment donné on choisit une série d'arguments et pas une autre. Il faudra analyser comment les différents arguments sont interconnectés entre eux, comment ils sont mis en série. Il faudra s'interroger sur la créativité lexicale, sur les représentations, sur les jeux de langage, sur l'usage des métaphores. Il convient de s'intéresser aux stratégies d'effets comme l'évidence, le déplacement et l'ambiguïté ${ }^{44}$ afin de rendre compte du lien entre une signification linguistique et un ensemble de comportements et pratiques. Il faudra enfin se demander comment ces rhétoriques transforment les logiques d'interaction, comment elles influencent les rapports de face-à-face. Il convient par ailleurs d'analyser comment ces discours structurent des opinions publiques, comment ils constituent des électorats. Pour qu'un discours politique ou sécuritaire puisse être efficace et constituer des opinions publiques, il faut qu'il s'adresse à ce que Murray Edelman appelle des " groupes cibles "55, c'est-à-dire des groupes choisis pour leur potentiel de sensibilité au problème proposé, et qu'il soit constitutif des opinions publiques. Pour analyser cet aspect de la question il faut s'intéresser à la construction sociale des " groupes cibles ". Selon Edelman, celle-ci se réfère aux caractéristiques culturelles et aux images populaires attribuées aux personnes et/ou groupes dont le comportement et le bien-être peuvent être affectés par le problème en question. Ces caractéristiques sont de l'ordre du normatif et sont évolutives. C'est à partir d'elles que 
sont réalisés des portraits des personnes et/ou groupes en termes négatifs ou positifs à travers l'usage d'un langage symbolique, de narratifs et de métaphores ${ }^{56}$. Il faudra également se rappeler que chaque pays a son répertoire propre de thématiques historiques et contextuelles, comme la peur de l'Islam pour la France et la crainte d'une hispanisation pour les Etats-Unis, qui ont un impact particulier sur les opinions publiques. Il faudra analyser comment les auteurs qui déploient ces arguments inventent des références sémantiques pour faire passer leurs messages. Par exemple, les discours accompagnant les lois sur l'immigration en France et aux Etats-Unis ont à un moment focalisé leurs arguments sur les droits sociaux. Il est intéressant d'indiquer que pour (dé)former les opinions publiques, ils ont effectué une modification sémantique en s'adressant non plus au citoyen comme c'était le cas jusqu'alors, mais au contribuable. Ils ont créé un climat de suspicion à l'encontre des immigrés bénéficiaires des droits sociaux qu'ils ont présenté comme des " fraudeurs " réels ou potentiels devenant " une charge " non seulement pour la collectivité mais surtout pour le contribuable à qui il incombait de supporter le coût en " payant de sa poche . ${ }^{57}$ ". Il faudra enfin porter une attention au fait communicationnel en envisageant les discours dans leur dimension interactionnelle, analyser les stratégies spécifiques, les contraintes et les supports utilisés.

Si l'approche d'Ole Waever ne permet pas de prendre en compte ces aspects de l'analyse du discours, on trouve, parmi les internationalistes, des auteurs qui, s'inspirant des philosophes comme Wittgenstein, intègrent différemment le langage dans leurs analyses. Ainsi, parmi les auteurs faisant partie de la tendance constructiviste de Critical security Studies, on peut citer les travaux de Karin Fierke. Elle emprunte à Wittgenstein son analyse linguistique afin de montrer comment un discours de sécurité a permis la construction de l'identité de l'OTAN et du Pacte de Varsovie $^{58}$. Reprenant les notions de contexte, de jeux de langage spécifiques à une culture et de métaphores développées par Wittgenstein, Karin Fierke étudie l'emploi des métaphores de la famille et de la maison dans la construction de l'idée de la sécurité en Europe (via l'analyse de l'OSCE, etc.) et montre comment on est passé de la métaphore de la famille à celle du club après la chute du mur de Berlin. Si l'analyse de Fierke permet d'étudier la signification que donnent les acteurs à leurs actions et si elle nous renseigne sur les contextes, la critique portée à Waever vaut pour elle, à savoir, s'appuyer sur une conception constructiviste de la réalité qui refuse à distinguer entre le monde perçu et le monde vécu. Quant à Beverly Crawford et Ronnie D. Lipshutz ${ }^{59}$, dans leur étude des discours de la guerre en ex-Yougoslavie, ils ne limitent pas leur analyse à la sémantique mais montrent comment ce qu'ils appellent les " entrepreneurs politiques " ont interprété le conflit bosniaque et ont développé des solutions alternatives nécessitant peu ou pas d'intervention des Etats de l'Union européenne dans ce conflit. Ils analysent le processus de politisation de l'ethnicité et de la religion et affirment que les " entrepreneurs politiques " pratiquent une politique de l'identité au détriment d'une politique de l'intérêt. La question que l'on pourrait leur poser est alors la suivante : pourquoi nous présenter une politique de l'identité et une politique d'intérêt comme deux politiques différentes, voire contradictoires? Les deux ne sontelles pas interdépendantes? N'y a-t-il pas derrière une politique identitaire, une politique d'intérêt?

18 A l'inverse de la plupart des auteurs qui se réfèrent à la philosophie du langage dans une perspective constructiviste, le propos de Michaël Dillon est tout à fait autre. Comme nous l'avons déjà indiqué, s'inspirant de la philosophie de Heidegger, il ne 
réduit pas le langage à une situation purement linguistique ou logique, car il s'agit pour lui d'une situation existentielle dont les phrases sont l'expression formelle. Considérant le parler comme un " existential ", le langage a trait aux fondements ontologiques. Ce qui explique l'emploi d'un L majuscule par Dillon dans son écriture du terme langage. La base du langage est dans la discursivité (Rede) qui est le propre de l'homme qui par son être est exposé à l'(in)sécurité de l'existence ${ }^{60}$. Ce qui implique que rien n'est sûr, rien n'est sans danger, y compris le langage. Pour montrer cette (in)sécurité existentielle, comme nous l'avons déjà mentionné, Dillon se réfère à l'étymologie et à la généalogie en arguant que les mots ne sont pas l'origine mais le chemin qui nous permet de saisir l'ambivalence de l'existence.

La sécurité dans sa dimension politique

Contrairement aux néoréalistes, qui ont plutôt tendance à ignorer la dimension politique de la sécurité en mettant l'accent sur sa dimension militaro-stratégique, les auteurs poststructuralistes ${ }^{61}$ et postmodernes proposent d'analyser la sécurité dans son rapport au politique. Comme l'affirment Michaël Williams et Keith Krause, en faisant ainsi, leur objectif n'est pas seulement de remettre en question la définition communément admise du politique, celle qui la lie à l'Etat et la souveraineté ainsi qu'à la conception de l'ordre qui en dérive, mais aussi de s'interroger sur les manières de la repenser dans un monde à la fois globalisé et fragmenté62. Le lien entre sécurité et politique peut être saisi de deux façons. D'une part, la sécurité est appréhendée comme étant constitutive du politique. De l'autre, elle est considérée comme articulant une activité de médiation ainsi que comme une praxis. Dans le premier cas, elle présuppose une menace, un danger ou une inimitié qui devient constitutive du politique. Elle définit alors la communauté politique en la distinguant et la protégeant de l'extérieur, de l'Autre, du différent. Dans le deuxième cas, comme le décrit Jef Huysmans, en séparant la vie de la mort, l'ordre du désordre, et se proposant d'accomplir une médiation entre elles, elle définit la place où apparaissent dans l'espace public les acteurs ainsi que les institutions politiques et sécuritaires qui pratiquent la médiation au nom de la communauté qu'ils représentent ${ }^{63}$. On peut dire que cet aspect de la question est celui qui est le moins traité en Relations Internationales. A l'exception de quelques auteurs comme Didier Bigo et Jean-Paul Hanon ${ }^{64}$, les internationalistes négligent d'analyser les agents de la sécurité comme les policiers, les militaires, les services secrets, et les institutions. En se référant aux travaux de Hobbes, de Machiavel et de Carl Schmitt, la plupart des études portant sur la sécurité se focalisent sur son aspect constitutif du politique en mettant en avant la question du danger, de la menace. Elles analysent la construction de la communauté politique, la question de l'ordre politique et celle de l'identité. Les auteurs postmodernes et poststructuralistes comme Michaël Dillon, R. B. J. Walker, David Campbell ont remis en cause l'approche classique de la communauté politique. Celle-ci s'appréhende au sens de population possédant des caractéristiques communes dont la survie est garantie à l'intérieur d'un territoire délimité. Ces caractéristiques sont souvent décrites en termes de communalité et d'homogénéité qui peuvent être menacées par le différent, l'autre, le désordonné. Critiquant l'aspect universalisant et homogénéisant des théories classiques de la communauté, ces auteurs ont mis en avant la question de la différence et de la fragmentation. Affirmant, à l'instar de David Campbell, que le lien entre menace, identité politique et sécurité n'est pas une évidence, ils ont fait ressortir son caractère paradoxal. En effet, si l'identité politique repose sur la menace de l'Autre, une politique sécuritaire qui se donne idéalement pour tâche d'éliminer celle-ci, ne finit-elle pas par 
supprimer l'identité politique, au cas où elle réalise effectivement son objectif ${ }^{65}$ ? Reprochant à ces théories d'identifier la communauté politique à l'Etat souverain, à l'instar de Simon Dalby et de R.B.J Walker, ils ont proposé de repenser la question du (des) sujet(s) de la politique ${ }^{66}$. Critiques vis-à-vis de l'ordonnancement classique établi entre Etat souverain, population, identité et sécurité, ces deux auteurs s'interrogent sur la manière de les analyser dans le cadre changeant de la nouvelle configuration mondiale. Ils remettent en cause la centralité de l'Etat comme le locus de l'action politique, son exclusivité comme acteur politique ainsi que la force normative et pratique de la souveraineté. R.B.J. Walker affirme que repenser la sécurité doit revenir à repenser le politique et lui envisager des localisations autres que dans l'Etat souverain. Cependant, sceptique vis-à-vis des approches globalistes qui proposent de passer du national au mondial voire au planétaire, il propose d'intégrer les travaux féministes et postcolonialistes qui interrogent la question des identités. Affirmant que le discours classique de la communauté revient à être un discours d'exclusion des subjectivités, il se donne comme tâche d'intégrer les autres subjectivités comme la classe sociale, la race, et le genre dans son analyse. Sceptique à l'égard des principes modernes d'autonomie et du sujet souverain, il critique l'idée selon laquelle ce n'est qu'à travers la souveraineté étatique qu'il est possible de résoudre les contradictions entre l'universel et le particulier, l'espace et le temps.

21 Ces interrogations touchent fondamentalement à un problème important qu'a soulevé la philosophie politique et morale dans les années quatre vingt. Il s'agit du passage de la conception kantienne du sujet (sujet autonome et universel) à une conception située du sujet, en insistant sur les concepts de différence et de spécificité. Affirmant que l'identité n'est jamais donnée une fois pour toutes, qu'elle n'est jamais fermée, et qu'elle est multiple et constamment en formation, les auteurs postmodernes se heurtent ici à une question fondamentale. C'est celle de la reconnaissance. Avec la multiplicité dans l'Un comment poser la question de la reconnaissance puisque l'identité la présuppose comme condition? Comment considérer les sujets à la fois comme ayant de multiples identités, comme étant différents mais en même temps égaux ? En ce qui concerne la sécurité, comment ne pas reproduire les cercles vicieux et les situations où plusieurs sujets et/ou entités font dépendre leur sécurité les unes des autres? Ces questions demandent une réflexion qui doit intégrer non seulement une dimension normative mais également pratique. La question de l'élimination de la souveraineté nécessite également une réflexion profonde. Comment peut-on concevoir un processus hors souveraineté ? Faut-il centrer l'interrogation sur sa disparition ou bien envisager un changement de sa signification? Sur cette question la position d'ole Waever est plus prudente. Reprenant Keohane qui affirme que " la souveraineté est moins une barrière définie en termes de territoire qu'un pouvoir de négociation au service d'une politique caractérisée par des réseaux transnationaux complexes ${ }^{67}$ ", Waever qualifie d'erronée la lecture de la souveraineté comprise par les libéraux comme un contrôle total effectif. En admettant qu'il puisse exister de nos jours " des processus hors souveraineté dans la cyber-économie, dans la réalité virtuelle, dans la réalité non territoriale d'innovation technologique ", il affirme que le système fondé sur la souveraineté continue de fonctionner selon sa propre logique ${ }^{68}$. Une des questions qui se pose ici est de savoir si on peut penser la sécurité en des termes qui ne présupposent pas un lien entre menace, politique et ordre. Ainsi David Campbell et Simon Dalby se demandent s'il est possible de concevoir la politique étrangère en terme d'une éthique de la responsabilité où les acteurs (sujets) ne seront pas présupposés 
comme étant autonomes mais dépendants les uns des autres. Pour cela, ils proposent de modifier les pratiques de l'international en éliminant la conception de la souveraineté comme contrôle total sur un territoire ${ }^{69}$. Ole Waever répond à cette question avec son concept de désécurisation ${ }^{70}$. Partant toujours de son approche linguistique, il affirme que la désécurisation apparaît quand les acteurs évitent d'énoncer un speech act sécuritaire dans des domaines politiques particuliers. Mais cependant, l'approche de Waever reste plus descriptive que normative. Se proposant d'élever le débat sur le plan normatif, Jef Huysmans reproche à Waever de ne pas éliminer de sa pensée une approche de la sécurité en termes de conflit et de guerre ${ }^{71}$. Il souligne que sa définition de sécurité n'est ni innovatrice, ni révolutionnaire, mais qu'elle s'inscrit bel et bien dans la vision du réalisme politique. En effet, comme Buzan, Waever part de l'acceptation de la sécurité comme une question de survie et la définit comme " étant structurée autour de la problématique de la guerre " et poursuit en écrivant " la guerre est essentiellement un phénomène inter-étatique dans un monde où les concepts de base sont la souveraineté, la défense et les frontières ${ }^{72}$ ". Selon Huysmans, afin de dépasser une logique de la guerre il faudra plutôt de se donner pour objectif le passage d'une conception du changement social en termes de violence à une conception en termes de changement pacifique. Comment éliminer de la sécurité l'idée de la violence, du conflit, de la menace? Mais dans ce cas, y a-t-il lieu de penser la sécurité ? Si oui comment l'envisager?

Ce parcours des diverses analyses a montré l'ambition des internationalistes contemporains de rompre avec la vision classique de la sécurité. Nous avons vu que pour ce faire ils élargissent leurs analyses à de nouvelles dimensions comme les dimensions normative, conceptuelle, descriptive, critique, linguistique, généalogique, etc., de la question de la sécurité. Mais aussi diverses, enrichissantes et intéressantes soient-elles, ces analyses manquent de traiter un aspect fondamental du processus de sécurisation. Elles ne traitent pas de la problématisation de la sécurité. Si certains auteurs comme Ole Waever évoquent parfois le " problème de sécurité73 ", ils ne nous disent pas pourquoi à un moment donné, un phénomène ou une question devient une question politique et comment une question politique se transforme en un problème et un enjeu de sécurité. C'est la question de la problématisation qui à notre avis constitue l'aspect le plus important de l'analyse du processus de la sécurisation. Pour la poser, en nous référant aux travaux d'Edelman, nous dirons qu'il convient d'analyser la mise en scène politique, les jeux politiques, les jeux bureaucratiques et les jeux médiatiques ${ }^{74}$. Il faut analyser le rôle des politiciens, des agents de la sécurité, des bureaucraties, des associations, des médias et étudier leurs interactions. Il faut voir comment ils construisent un problème dont le caractère politique réside dans l'amalgame des questions diverses dans les traits d'un ennemi, dans l'ambiguïté créée par les dispositifs narratifs et dans la non-résolution des controverses soulevées par différentes significations. Il faut s'interroger sur la fonction politique de ces ambiguïtés et ambivalences. Il faut enfin analyser leurs effets sur les opinions publiques. 


\section{NOTES}

1. Arnold Wolfers, Discord and Collaboration, Essays on International Politics, Baltimore, John Hopkins University Press, 1962. John Herz, " Idealist Internationalism and the Security Dilemma ", World Politics, 2 (2), 1950. Harold D. Laswell, World Politics and Personal Insecurity, New York, Free Press, 1935, rééd. 1965.

2. Voir la contribution quasi auto-biographique de Ken Booth, " Security and Self : Reflections of a Fallen Realist ", in Keith Krause \& Michael C.Williams (eds), Critical Security Studies,University of Minnesota Press, Borderlines vol. 8, 1997, pp. 83-119. 3. Michael Dillon, Politics of Security : Towards a Political Philosophy of Continental Thought, London, New York, Routledge, 1996, p. 12.

4. Ibid., pp. 13 et 17.

5. Voir Jef Huysmans, " Security! What do you mean? " in European Journal of International Relations, vol. 4 (2), 1998, pp. 226-255 ; cf. " Troubler et inquiéter, les discours du désordre international " (ss. la dir. de Didier Bigo et Jean-Yves Haine), Cultures \& Conflits, n 19/20, 1995 ; " The Mobius Ribbon ", ISA, Toronto, 1997.

6. Brighton, Harvester, 1983, réédition en 1993.

7. Didier Bigo, " L'Europe de la sécurité intérieure : penser autrement la sécurité ", in Anne-Marie Le Gloannec (dir), Entre union et nations, Presses de Sciences Po, Paris, 1998, pp. 65-67.

8. Sur l'élargissement et l'approfondissement, voir K. Krause \& M. C. Williams (eds), op.cit.

9. Les auteurs qui se regroupent sous ce label se sont initialement réunis au York Center for International and Strategic Studies (YCISS) à Toronto.

10. Voir la préface de M. C. Williams et de K. Krause, " Preface : Toward Critical Security Studies ", op. cit., pp. vii-xxi.

11. Ole Waever, op. cit., p. 13., Dillon, op. cit., p. 122.

12. Simon Dalby, " Contesting an Essential Concept : Reading the Dilemmas in Contemporary Security Discourse " in Critical Security Studies, op. cit., p. 6.

13. Voir W. B. Gallie, " Essentially Contested Concept " in Max Black (ed), The Importance of Language, Englewood Cliffs, N. J., Prentice Hall, 1962 ; William Connolly, The Terms of Political Discourse, Princeton, N.J., Princeton University Press, 1983.

14. Ole Waever, op. cit., p. 87.

15. Jef Huysmans, op. cit., p. 134.

16. Jef Huysmans, op. cit., p. 136.

17. Michael Dillon, op. cit., p. 118.

18. Ibid., p. 120.

19. Ibid. p. 121.

20. On trouve cette forme duale de transcription des termes à signification ambiguë dans l'œuvre de Heidegger, par exemple le terme Da-sein (l'homme caractérisé par le fait d'être-là).

21. On trouve cette forme duale de transcription des termes à signification ambiguë dans l'œuvre de Heidegger, par exemple le terme Da-sein (l'homme caractérisé par le fait d'être-là).

22. Ibid., pp. 78, 120. 
23. James Der Derian, " The Scriptures of Security ", Mershon International Studies Review, vol. 42, suppl.1, May 1998, p. 120.

24. Ole Waever, op. cit., pp. 13, 14. Voir aussi le dernier texte du livre " Self-referential concepts of security as an instrument for reconstruction of an open-ended realism in IR ", pp. 347-372.

25. Ibid.

26. Ole Waever, Concepts of Security, Institute of Political Sciences, University of Copenhagen, 1997, p. 17.

27. Buzan avait défini la sécurité sociétale comme " les conditions acceptables dans lesquelles on peut prévoir l'évolution des modèles traditionnels du langage, de la culture, de l'identité nationale et de la coutume ", Peoples, States and Fear, op. cit., p. 17.

28. Ole Waever, "Societal security : the concept " in O. Waever, B. Buzan, M. Kelstrup, P. Lemaître (eds.), Identity Migration and the New Security Agenda in Europe, New York, St Martin's Press, 1993.

29. Ole Waever, "Insécurité et identité. Une dialectique sans fin ", in Anne-Marie Le Gloannec (dir), Entre Union et Nations, op.cit., p. 97.

30. Ibid., p. 95.

31. Herder voit les nations comme des unités naturelles et affirme que l'homme se définit par rapport au groupe, à la culture, à la nation auxquels il appartient et dans lesquels il peut s'épanouir. Selon lui, l'expression de la nation au sein de l'Etat n'est pas nécessaire. Cf. Dictionnaire de la pensée politique, Hatier, Paris, 1989. Pour développer son approche, Waever cite les travaux d'Isiah Berlin. Voir Societal Security : the Concept, op. cit., p. 34.

32. Voir les critiques de Didier Bigo, "L'Europe de la sécurité intérieure. Penser autrement la sécurité ", op.cit.

33. Voir Ayse Ceyhan, " Migrants as A Threat : A Comparative Analysis of a Securitarian Discourse : France and the United-States ", in V.Gray (ed.), A European Dilemma. Immigration, Citizenship and Identity in Western Europe, Providence, Oxford, Berghahn Books (à paraître).

34. " Securitization and Desecuritization ", in Ronnie Lipshutz (ed.), On Security, Columbia University Press, 1995.

35. Ole Waever, op. cit., p. 13.

36. Voir Manuel Maria Carrilho, " La pragmatique ou l'action par le langage : Austin et Searle ", in Michel Meyer (dir.), La philosophie anglo-saxonne, Paris, PUF, 1994, pp. 368-386.

37. J.L.Austin, Quand dire c'est faire, Paris, Seuil, 1970, pp. 41-42.

38. " Securitization and Desecuritization " op. cit., pp. 46-86 et " Insécurité, identité. Une dialectique sans fin ", op. cit., p. 99.

39. Il semble que dans ses travaux antérieurs Waever était plus enclin à effectuer une analyse du discours en examinant les textes des acteurs spécifiques comme son étude du discours sécuritaire des quatre principales têtes pensantes du SPD allemand en 1989 : Bahr, Eppler, Ehmke et Voyt. Ses conclusions sont intéressantes. Il écrit : " finalement ces auteurs disent pratiquement la même chose mais en déployant des arguments différents, parfois même en se contredisant ". Voir " Ideologies of Stabilization - Stabilization of Ideologies. Reading German Social Democrats " in V. Harle \& P.Sivonen (eds), Europe in Transition, Politics and Nuclear Strategy, London, Pinter, 1989. Waever a repris ce texte dans Concepts of Security, op. cit. pp. 152-183. 
40. " In Search of " security " : Report on a Journey among concept (résumé), in Concepts of Security, op. cit., p. 8.

41. Pour une distinction entre une linguistique de l'énonciation et une linguistique ou analyse du discours, voir " Les analyses du discours en France ", sous la direction de Dominique Maingueneau, Langages, $\mathrm{n}^{\circ}$ 117, Mars 1995, p. 7.

42. Référence qu'il énonce rapidement et souvent en note de bas de page, par exemple dans Concepts of Security, p. 14, note 14, ou dans la reprise du "Securititization, Desecuritization " dans le même livre, note 30, p. 249 etc.

43. Jacques Derrida, " Signature, Event, Context ", Glyph, vol. 1, 1977. Ce texte a été initialement publié dans Marges de la philosophie, Paris Editions de Minuit, 1972 sous le titre " Signature, événement, contexte ". Dans le numéro suivant de Glyph, Searle a critiqué les commentaires de Derrida dans son article intitulé "Reiterating the Differences " qui a été traduit en français sous le titre Pour réitérer les différences. Réponse à Derrida, Combas, Editions de l'éclat, 1991. Searle a ensuite exposé ses critiques dans " The Word Turned Upside Down, The New York Review of Books, 27 novembre 1977, qui a été traduit en français sous le titre Déconstruction. Le langage dans tous ses états, Combas, Editions de l'éclat, 1992.

44. John Searle est l'auteur de nombreux ouvrages dont Speech Act (1969) traduit en français sous le titre de Les actes du langage, Paris, Hermann, 1972, et The Construction of Social Reality, The Free Press, 1995.

45. John Searle, Pour réitérer les différences, op. cit.

46. J. Derrida, Marges de la philosophie, op.cit., p. 376.

47. Ibid., p. 383.

48. Ibid. p. 381, et aussi pp. 382-390.

49. Voir Réitérer les différences et la postface de Joëlle Proust, pp. 25-29.

50. John Searle, Déconstruction. Le langage dans tous ses états, op. cit., pp. 8-9.

51. Ibid.

52. Albert O. Hirschman, Deux siècles de rhétorique réactionnaire, Paris, Fayard, 1991, p. 21.

53. Pour une analyse des arguments rhétoriques, voir Ayse Ceyhan, " Migrants as a Threat.. ", op. cit.

54. Voir M. Souchard, S. Wahnich, I. Cuminal, V. Wathier, Le Pen. Les mots. Analyse d'un discours d'extrême-droite, Le Monde Editions, 1997, p. 22.

55. Murray Edelman, The Symbolic Uses of Politics, Urbana, University of Illinois Press, 1964.

56. Voir Anne Schneider et Helen Ingram, " Social Construction of Target Populations. Implications for Politics and Policy ", American Political Science Review, vol. 87, $\mathrm{n}^{\circ} 2$, June 1993.

57. Voir Ayse Ceyhan, " Migrants as a Threat... ", op. cit. et aussi Kitty Kalavita, The Politics of Immigration : " Balanced-Budget Conservatism " and the Symbolism of Prop. 187, University of California, Irvine, 1996.

58. Karin M. Fierke, " Changing Words of Security ", in Critical Security Studies, op. cit., pp. 223-255.

59. B.Crawford et R.D.Lipshutz, " Discourses of War : Security and the Case of Yougoslavia ", in Critical Security Studies, op. cit., pp. 149-187.

60. Voir Dillon, op. cit., p.131.

61. Comment ces appellations sont-elles forgées ? Sont-elles des auto-identifications faites par les intéressés eux-mêmes ou bien sont-elles le résultat de désignations faites 
par des autres ? Ces questions méritent d'être étudiées. En attendant, je citerai une définition du postructuralisme fournie par Waever : " Les post-structuralistes soulignent que le système de signes n'est jamais complètement fermé et que le sens par conséquent n'est jamais fini ", Insécurité, identité : une dialectique sans fin, op. cit., p. 116.

62. M. Williams, K. Krause, " Preface : Towards a Critical Security Studies ", op. cit., pp. $\mathrm{x}, \mathrm{xi}$.

63. Voir Jef Huysmans, " Security! What do you mean ? " op. cit., p. 244.

64. Jean-Paul Hanon, " L'armée veille à El Paso ", Le Monde Diplomatique, novembre 1996. Thèse en préparation.

65. David Campbell, Writing Security, United States Foreign Policy and the Politics of Identity, Minneapolis, University of Minnesota Press, 1992.

66. Simon Dalby, op. cit. ; R. B. J. Walker, The Subject of Security, in Critical Security Studies, op. cit. pp. 61-82.

67. Robert O. Keohane, " Hobbes, Dilemma and International Change in World Politics : Sovereignty in International Society ", in H.-H.Holm, G.Soresten (eds.), Whose World Order? Uneven Globalization and the End of the Cold War, Boulder, Colorado, Westview Press, 1995, p. 177.

68. Ole Waever, " Insécurité, identité... ", op. cit., p. 107.

69. Simon Dalby, op.cit., David Campbell, Politics Without Principle. Sovereignty, Ethics and the Narrative of the Gulf War, London, Lynne Rienner, 1993.

70. Voir Ole Waever " Securitization and Desecuritization ", in Ronnie L. LIpschutz

(ed.), On Security, New York, Columbia University Press, 1995, pp. 46-87.

71. Jef Huysmans, op.cit.

72. Ole Waever " Conflict of Vision - Visions of Conflict ", op. cit., p. 86. Pour une critique, voir Jef Huysmans, " The Question of the Limit : the Name of the Migrant ; Desecuritisation and the Aesthetics of Rupture in Political Realism ",(à paraître).

73. Par exemple dans Concepts of Security, op. cit. p. 67.

74. Murray Edelman, Pièces et règles du jeu politique, Paris, Seuil, 1991.

\section{INDEX}

Mots-clés : Relations Internationales, théories de la sécurité 